\title{
Efecto de un concentrado de inicio y cerca gazapera sobre los parámetros productivos en cuyes lactantes
}

\author{
Effect of a starter concentrate and a litter fence on the productive performance \\ of lactating guinea pigs
}

\author{
Carmen Soto Mateo ${ }^{1}$, Víctor Hidalgo Lozano ${ }^{1,2}$, Elva Ríos Ríos ${ }^{1}$
}

\section{Resumen}

\begin{abstract}
El objetivo del presente trabajo fue estudiar el uso de concentrado de inicio y cercas gazaperas en cuyes lactantes hasta el deteste (15 días). Los tratamientos fueron: TI, Concentrado convencional (CC) y sin cerca gazapera; Control; TII, CC + cerca gazapera; TIII, CC + concentrado de inicio + cerca gazapera. Se evaluó la ganancia de peso, consumo de alimento y mortalidad al destete. Se utilizaron nueve pozas de $1 \mathrm{~m}^{2}$ con 5 hembras y 1 macho por poza y con cercas gazaperas (forma triangular con un área de $0.125 \mathrm{~m}^{2}$ ) en su interior (TII y TIII) donde se ubicaron comederos de arcilla para el concentrado de inicio (20\% de proteína y $2781 \mathrm{kcal} / \mathrm{kg}$ de energía digestible). El concentrado convencional (comercial) para los adultos aportó $18.7 \%$ de proteína y $2888 \mathrm{kcal} / \mathrm{kg}$ de energía digestible. La ganancia de peso al destete fue de $10.3,9.5$ y $9.3 \mathrm{~g} / \mathrm{d}$ para T I, II y III, respectivamente, sin diferencia significativa entre ellos. El consumo de concentrado de las crías fue similar entre TII (19.4 g/d) y TIII ( $18.4 \mathrm{~g} / \mathrm{d})$ y el porcentaje de mortalidad al destete fue $11.5,13.9$ y $8.0 \%$ para T I, II y III, respectivamente.
\end{abstract}

Palabras clave: gazapo, Cavia porcellus, cerca gazapera, parámetros productivos

\section{Abstract}

The aim of this study was the use of starter concentrate and litter fences in lactating guinea pigs until weaning (15 days). The treatments were: TI, Conventional concentrate (CC) and without litter fence; Control; TII, CC + litter fence; TIII, CC + start concentrate + litter fence. Body weight gain, feed intake and mortality at weaning were evaluated.

\footnotetext{
${ }^{1}$ Universidad Nacional Agraria La Molina, Lima, Perú

${ }^{2}$ E-mail:vhidalgo@lamolina.edu.pe
}

Recibido: 5 de julio de 2019

Aceptado para publicación: 9 de junio de 2020

Publicado: 11 de agosto de 2020 
Nine $1 \mathrm{~m}^{2}$ pits with 5 females and 1 male per pit and litter fences (triangular shape with an area of $0.125 \mathrm{~m}^{2}$ ) were used (TII and TIII) where clay feeders for the starting concentrate were located $(20 \%$ protein and $2781 \mathrm{kcal} / \mathrm{kg}$ of digestible energy). The conventional (commercial) concentrate for mothers provided $18.7 \%$ protein and $2888 \mathrm{kcal} / \mathrm{kg}$ of digestible energy. The body weight gain at weaning was $10.3,9.5$ and $9.3 \mathrm{~g} / \mathrm{d}$ for T I, II and III, respectively, with no significant difference between them. The consumption of concentrate of the offspring was similar between TII $(19.4 \mathrm{~g} / \mathrm{d})$ and TIII $(18.4 \mathrm{~g} / \mathrm{d})$ and the percentage of mortality at weaning was $11,5,13.9$ and $8.0 \%$ for T I, II and III, respectively.

Key words: offspring, guinea pig, Cavia porcellus, litter fence, productive parameters

\section{INTRODUCCIÓN}

Las instalaciones e implementos para la crianza de cuyes (Cavia porcellus) deben ser diseñadas para mejorar su productividad y mantener un nivel de tecnología competitivo. El rango de temperatura debe estar entre 15 y $18{ }^{\circ} \mathrm{C}$, aunque el cuy es tolerante al frío (Solórzano, 2014; Huamani, 2017). Por otro lado, Chauca e Higaonna (1998) indican que fuentes de calor en épocas de frío intenso reducen la mortalidad en un $7 \%$.

El uso de cerca gazapera en la época de parición brinda un ambiente especial a las crías, protegiéndolas de los golpes y aplastamiento por las madres y permite que dispongan de alimento exclusivo sin competencia con los adultos (Chauca et al., 1994, Chauca e Higaonna, 1998), obteniendo una mayor tasa de sobrevivencia en las crías (Caycedo, 2000). En este sentido, el INIA y CIID (1994) reportan que la mortalidad de gazapos de 70$80 \mathrm{~g}$ de peso vivo en crianza sin cerca gazapera fue de $23 \%$.

Los gazapos de 15 días de edad consumen concentrado si es suministrado a temprana edad. La edad propicia para el destete puede variar con las condiciones de crianza. Así Moreno (1989) recomienda hacerlo a los 21 días, mientras que Aliaga (1993) recomienda entre los 10 días y 15 días de lactación. Por otro lado, Sarria (2014) recomienda realizar el destete a los 14 días para cuyes mejo- rados, cuando los gazapos están alrededor de $200 \mathrm{~g}$ de peso.

Moreno (1989) menciona que los cuyes en crecimiento pueden incrementar de 4 a 10 g diarios, mientras que Chauca y Ordoñez (1995) indican que los gazapos duplican su peso hasta el destete; siendo los incrementos mínimos los primeros cinco días, alcanzando hasta $10.2 \mathrm{~g}$ /día a partir del sexto día. Por otro lado, Peruano (1999) observó que los incrementos de peso varían según tamaño de camada reportando valores de 12.0, 10.0, 8.9, 8.1, $6.6 \mathrm{~g}$ /día con partos de 1, 2, 3, 4 y 5 crías, respectivamente. Con respecto a la mortalidad en el periodo del nacimiento al destete y bajo un sistema de empadre permanente, se reportan tasas entre 6.8 y $13.6 \%$ en cuyes con raciones de inicio con y sin inclusión de forraje verde, respectivamente, sin diferencias entre tratamientos (Mamani, 2016) y entre 9 y $29 \%$ (Mendoza, 1980).

En trabajos con suplementación, Sarria et al. (2019) reportaron ganancias de peso de 12.6 y $13.0 \mathrm{~g} /$ día en cuyes alimentados con raciones de energía digestible (ED) de 2700 y $2900 \mathrm{kcal} /$ $\mathrm{kg}$ y sin inclusión de forraje verde, respectivamente. Por otro lado, Hancco (2017) evaluó el efecto de cuatro densidades nutricionales, utilizando raciones con niveles de energía metabolizable(EM) de 2550, 2700, 2850 y 2920 $\mathrm{kcal} / \mathrm{kg}$ y de proteína de $17.70,18.74,19.78 \mathrm{y}$ $20.82 \%$, respectivamente, sin encontrar diferencias significativas para ganancia de peso $y$ consumo de alimento en cuyes al destete. 
Con la finalidad de hacer un aporte a los sistemas de crianza de cuyes, el presente trabajo tuvo como objetivo evaluar el uso de un concentrado de inicio y cerca gazapera sobre el incremento de peso y el porcentaje de mortalidad en crías de cuyes al destete.

\section{Materiales y Métodos}

El presente trabajo de investigación se realizó en las instalaciones de la Granja de Animales Menores del Programa de Investigación en Carnes de la Universidad Nacional Agraria La Molina (UNALM), ubicada en la provincia y departamento de Lima, Perú. Las muestras de alimento concentrado y forraje se analizaron en el Laboratorio de Evaluación Nutricional de Alimentos del Departamento Académico de Nutrición, Facultad de Zootecnia, UNALM.

El experimento se realizó en un galpón de material noble de $6.20 \mathrm{~m}$ de ancho, $35 \mathrm{~m}$ de largo y $2.80 \mathrm{~m}$ de altura. Se utilizaron nueve pozas experimentales de $1.0 \mathrm{~m}$ de largo por $1.0 \mathrm{~m}$ de ancho y $0.37 \mathrm{~m}$ de alto, con un área total de $1.0 \mathrm{~m}^{2}$ por poza. El piso de cemento de cada poza fue cubierto con una capa de coronta de maíz como material de piso absorbente. En cada poza se colocó un comedero de arcilla con capacidad para $500 \mathrm{~g}$ de alimento concentrado convencional para las madres y otro pequeño de $60 \mathrm{~g}$ de capacidad para concentrado de inicio dentro de la cerca gazapera con excepción del tratamiento control. Las cercas gazaperas de forma triangular fueron ubicadas en una esquina apoyadas sobre las paredes de la poza a una altura de $26 \mathrm{~cm}$. La cerca medía $50 \mathrm{~cm}$ de lado y $70 \mathrm{~cm}$ de hipotenusa, lo que hacia un área de $0.125 \mathrm{~m}^{2}$, exclusiva para los gazapos lactantes.

Se utilizaron 45 hembras de $2^{\circ}$ y $3^{\circ}$ parto $(5$ por poza) del genotipo Cieneguilla y 9 machos (1 por poza), procedentes de la Granja de Cuyes de Cieneguilla de la UNALM. Las crías permanecieron con sus padres hasta el destete a los 15 días de edad. El concentrado tipo comercial de los padres contenía un aporte proteico de $18.8 \%$ y $2781 \mathrm{kcal} / \mathrm{kg}$ de energía digestible (ED) y para las crías fue un concentrado de inicio con $20.0 \%$ de proteína y $2888 \mathrm{kcal} / \mathrm{kg}$ de ED (Cuadro 1). Los concentrados fueron preparados siguiendo las recomendaciones del Comité de Nutrición del National Research Council (NRC, 1995). Así mismo, se suministró $1.5 \mathrm{~kg}$ de maíz chala verde por poza ( $250 \mathrm{~g} /$ cuy) diariamente. La composición química nutricional del maíz chala se muestra en el Cuadro 2.

Se evaluaron tres tratamientos con tres repeticiones cada uno ( 5 hembras gestantes y 1 macho por repetición). Los cuyes fueron distribuidos al azar en los siguientes tratamientos, TI: Concentrado convencional (CC) y sin cerca gazapera, TII: CC y con cerca gazapera, y TIII: $\mathrm{CC}+$ concentrado de inicio y con cerca gazapera. El agua se administró a discreción en pocillos de porcelana de $200 \mathrm{ml}$ de capacidad.

Se evaluó la ganancia de peso vivo, calculada como la diferencia entre el peso al destete y el peso al nacimiento de cada cría. El consumo de alimento para TII y TIII fue calculado semanalmente mediante la diferencia de alimento suministrado y el residuo. No se controló el consumo de los gazapos en TI (con dieta convencional), dado que no se utilizó la cerca gazapera. La mortalidad se determinó en base al conteo de los gazapos muertos, expresado en porcentaje. Se registró el número de nacidos muertos por camadas y de crías muertas por tratamiento.

Los datos se procesaron utilizando un Diseño Completo al Azar con tres tratamientos y tres repeticiones cada uno para la evaluación de ganancia de peso vivo. La comparación de promedios se hizo mediante la prueba de Duncan con una probabilidad de 5\%. Para la evaluación del consumo de alimento entre TII y TIII se utilizó la prueba t-Student pareada con dos colas con una probabilidad de $5 \%$. 
Cuadro 1. Composición porcentual y nutricional de los concentrados para los cuyes (Base seca)

\begin{tabular}{lcc}
\hline Ingredientes & Convencional & Inicio \\
\hline Afrecho de trigo & 72.00 & 61.90 \\
Torta de soya & 12.00 & 18.67 \\
Forraje seco de maíz & 12.00 & 10.50 \\
Azúcar rubia & - & 4.00 \\
Maíz grano molido & - & 2.00 \\
Carbonato de calcio & 3.02 & 2.00 \\
Fosfato monodicálcico & 0.43 & 0.40 \\
Olaquinidox & 0.20 & 0.20 \\
Sal común & 0.18 & 0.15 \\
Rovimix Stay - 35 & 0.10 & 0.10 \\
Metionina & 0.07 & 0.09 \\
\hline Total & 100 & 100 \\
\hline
\end{tabular}

Cuadro 2. Composición química proximal del concentrado (Convencional e Inicio) y del maíz chala (base fresca)

\begin{tabular}{lccc}
\hline Nutriente & $\begin{array}{c}\text { Convencional } \\
\%\end{array}$ & $\begin{array}{c}\text { Inicio } \\
\%\end{array}$ & $\begin{array}{c}\text { Maíz chala } \\
\%\end{array}$ \\
\hline Humedad & 10.04 & 10.02 & 76.61 \\
Proteína total & 18.90 & 20.55 & 1.92 \\
Extracto etéreo & 3.83 & 3.92 & 0.29 \\
Fibra cruda & 9.00 & 6.99 & 5.89 \\
Ceniza & 7.12 & 6.30 & 1.41 \\
Extracto libre de & 51.64 & 52.22 & 13.88 \\
nitrógeno (ELN) & & & \\
\hline
\end{tabular}

Fuente: Laboratorio de Evaluación Nutricional de Alimentos - UNALM 
Cuadro 3. Peso al nacimiento y destete y ganancia de peso en cuyes con o sin concentrado de inicio y con y sin gazapera

\begin{tabular}{ccccc}
\hline & $\begin{array}{c}\text { Peso al } \\
\text { nacimiento } \\
(\mathrm{g})\end{array}$ & $\begin{array}{c}\text { Peso al destete } \\
(\mathrm{g})\end{array}$ & $\begin{array}{c}\text { Ganancia de peso } \\
\text { total }(\mathrm{g})\end{array}$ & $\begin{array}{c}\text { Ganancia de } \\
\text { peso } \\
(\mathrm{g} / \text { día })\end{array}$ \\
\hline I & 161 & 326 & $154.7^{\mathrm{a}}$ & $10.3^{\mathrm{a}}$ \\
II & 160 & 304 & $143.8^{\mathrm{a}}$ & $9.5^{\mathrm{a}}$ \\
III & 161 & 307 & $139.5^{\mathrm{a}}$ & $9.3^{\mathrm{a}}$ \\
\hline
\end{tabular}

a Valores dentro de columnas con igual superíndice indican que no existen diferencias significativas

TI: Concentrado convencional (CC) y sin cerca gazapera, TII: CC y con cerca gazapera, TIII: CC + concentrado de inicio y con cerca gazapera

\section{Resultados y Discusión}

El peso al nacimiento fue similar entre tratamientos, dado que las condiciones de manejo y alimentación durante la gestación fueron similares para todas las madres. No obstante, tampoco hubo diferencias significativas en el peso al destete (Cuadro 3). Asimismo, tampoco se encontró diferencia significativa entre tratamientos en la ganancia de peso vivo entre el nacimiento y el destete a los 15 días (Cuadro 3), aunque con una tendencia a mayor ganancia de peso en TI. Similar comportamiento del cuy con el uso de raciones con dos niveles de energía, y con y sin inclusión de forraje verde, fueron reportados por Sarria et al. (2019); en tanto que Peruano (1999) reportó ganancias de peso superiores a los $8.81 \mathrm{~g} /$ día.

En lo que respecta al consumo de alimento, en el Cuadro 4 se muestran los promedios de consumo de alimento del nacimiento al destete, en el cual se observa que no existen diferencias significativas entre tratamientos.
Cuadro 4. Consumo promedio de alimento del nacimiento al destete en cuyes con o sin concentrado de inicio y con y sin gazapera

\begin{tabular}{lc}
\hline Tratamiento $^{1}$ & $\begin{array}{c}\text { Consumo de } \\
\text { alimento }(\mathrm{g} / \mathrm{d})\end{array}$ \\
\hline II & $19.43^{\mathrm{a}}$ \\
III & $18.43^{\mathrm{a}}$ \\
\hline a Valores en columnas con igual superíndice \\
indican que no existen diferencias \\
significativas \\
${ }^{1}$ TII: Concentrado convencional (CC) y con \\
cerca gazapera, TIII: CC + concentrado de \\
inicio y con cerca gazapera
\end{tabular}

El consumo de alimento al destete fue estadísticamente similar entre TII y TIII, y similares a los reportados por Correa (1988) y Moreno (1989), quienes reportan variaciones entre 8 y 30 g/día. En el experimento, asimismo, se observó que las crías preferían consumir el concentrado convencional de las 
Cuadro 5. Mortalidad al nacimiento y al destete en cuyes con o sin concentrado de inicio y con o sin gazapera

\begin{tabular}{cccccccc}
\hline Trat. & $\begin{array}{c}\text { Crías } \\
\text { nacidas } \\
(\mathrm{n})\end{array}$ & $\begin{array}{c}\text { Nacidos } \\
\text { muertos } \\
(\mathrm{n})\end{array}$ & $\begin{array}{c}\text { Mortalidad } \\
\text { al } \\
\text { nacimiento } \\
(\%)\end{array}$ & $\begin{array}{c}\text { Nacidos } \\
\text { vivos } \\
(\mathrm{n})\end{array}$ & $\begin{array}{c}\text { Muertos } \\
\text { al destete } \\
(\mathrm{n})\end{array}$ & $\begin{array}{c}\text { Destetado } \\
(\mathrm{n})\end{array}$ & $\begin{array}{c}\text { Mortalidad } \\
\text { al destete } \\
(\%)\end{array}$ \\
\hline I & 30 & 4 & 13.3 & 26 & 3 & 23 & 11.5 \\
II & 41 & 5 & 12.2 & 36 & 5 & 31 & 13.9 \\
III & 28 & 3 & 10.7 & 25 & 2 & 23 & 8.0 \\
\hline Total & 99 & 12 & 12.1 & 87 & 10 & 77 & 11.5 \\
\hline
\end{tabular}

TI: Concentrado convencional (CC) y sin cerca gazapera, TII: CC y con cerca gazapera, TIII: CC + concentrado de inicio y con cerca gazapera

madres, posiblemente como un comportamiento natural de imitar a los padres. Este consumo de concentrado por las crías en los comederos de las madres no fue medido, pero se presume que debió haber sido mayor en las pozas de TIII, pues el concentrado de inicio ofrecía un nivel energético y proteico mayor, lo que podría haber reducido este consumo (Aliaga, 1993).

El aporte de nutrientes, tanto de la leche materna como del concentrado convencional y de inicio, satisface los requerimientos recomendados por la NRC (1995); sin embargo, no se midió el consumo de forraje (maíz chala), el cual podría causar una variación en el consumo de fibra. No obstante, el rango recomendado de fibra en la dieta de cuyes es de 6 a 18\% (Aliaga, 1993). Entre ambas raciones, la de inicio tiene un mejor nivel de nutrientes excepto la fibra, lo que puede haber desfavorecido su consumo y aprovechamiento como mencionan Moreno (1989) y Aliaga (1993).
La mortalidad al nacimiento fue de $12.1 \%$ (Cuadro 5), valores similares a los reportados por Moreno (1989) y Mamani (2016), en tanto que, Peruano (1999) obtuvo una mortalidad promedio al nacimiento de $4.35 \%$.

La mortalidad al destete fue de 11.5, 13.9 y $8.0 \%$ para los tratamientos I, II y III, respectivamente (Cuadro 5). Al respecto, Peruano (1999) reporta que la mortalidad durante la lactación fue de $14.55 \%$ en el $3^{\circ}$ parto, siendo mayor la mortalidad en las crías de bajo peso. Similar respuesta al uso de cerca gazapera fue observada en el trabajo del INIA y CIID (1994) con una densidad de 1:6 cuyes y con tasas de mortalidad de $7.1 \mathrm{y}$ $14.4 \%$ con y $\sin$ cerca gazapera, respectivamente.

\section{Conclusiones}

- La ganancia de peso y el consumo de concentrado al destete a los 15 días de edad en cuyes no se afectó por el em- 
pleo de una dieta de inicio apara las crías ni por el uso de un cerco gazapero.

\section{Literatura Citada}

1. Aliaga RL. 1993. Crianza de cuyes. Instituto de investigación Agraria. DGIA. TTA/USAID. Serie Manual No 9 -93. Lima, Perú. 97 p.

2. Caycedo A. 2000. Experiencias investigativas en la producción de cuyes. Contribución al desarrollo técnico de la explotación. Pasto, Colombia: Univ. de Nariño. 323 p.

3. Correa R. 1988. La crianza del cuy. Colombia: Ministerio de Agricultura e Instituto Colombiano Agropecuario. ICASENA. 47 p.

4. Chauca L, Higaonna R. 1998. Producción de cuyes, manejo de reproductores. Folleto R.I. No 2-98. Instituto Nacional de Investigación Agraria. 27 p.

5. Chauca L, Ordoñez R. 1995. Caracterización de la lactación en cuyes. En: XVIII Reunión de la Asociación Peruana de Producción Animal - APPA. Lambayeque, Perú.

6. Chauca L, Rojas S, Calapuja A. 1994. Lactación de cuyes: utilización de cercas gazaperas. En: XVII Reunión de la Asociación Peruana de Producción Animal. Lima, Perú.

7. Hancco DC. 2017. Efecto de cuatro densidades nutricionales en el destete precoz (7 días) de cuyes (Cavia porcellus). Tesis de Ingeniero Zootecnista. Cusco, Perú: Univ. Nacional de San Antonio Abad de Cusco. 112 p.

8. Huamaní EN. 2017. Engorde de cuyes (Cavia porcellus) en pozas y jaulas con peso emparrillado de plástico. Tesis de Ingeniero Zootecnista. Lima, Perú: Univ. Nacional Agraria La Molina. 45 p.

9. [INIA] Instituto Nacional de Investigación Agraria, [CIID] Centro Internacional de Investigación para el
Desarrollo. 1994. Proyecto Sistemas de Producción de Cuyes. Tomo II. [Internet]. Disponible en: https://idl-bncidrc.dspacedirect.org/bitstream/handle/ 10625/35272/104403_v2.pdf?-sequen$\mathrm{ce}=4 \&$ isAllowed $=\mathrm{y}$

10. Mamani LTD. 2016. Evaluación de dos niveles de energía y dos sistemas de alimentación en dietas altas en fibra durante la reproducción de cuyes (Cavia porcellus). Tesis de Ingeniero Zootecnista. Lima, Perú: Univ. Nacional Agraria La Molina. $128 \mathrm{p}$.

11. Mendoza S. 1980. Estudio de productos no tradicionales para la alimentación de cuy. Tesis de Grado: Lima, Perú: Univ. Nacional Agraria La Molina. $87 \mathrm{p}$.

12. Moreno AE. 1989. Producción de cuyes. $2^{\circ}$ ed. Lima, Perú: Univ. Nacional Agraria La Molina. $132 \mathrm{p}$.

13. [NRC] National Research Council. 1995. Nutrient requirement of laboratory animals. $4^{\text {th }}$ Rev. Ed. Washington DC: NRC. 192 p. doi.org/10.17226/4758

14. Peruano DM. 1999. Evaluación de la vida productiva y reproductiva del cuy hembra (Cavia porcellus) en cuatro partos utilizando empadre continuo. Tesis de Ingeniero Zootecnista. Lima, Perú: Univ. Nacional Agraria La Molina. $88 \mathrm{p}$.

15. Sarria J, Vergara V, Cantaro J, Alejandro P. 2019. Evaluación de niveles de energía digestible en dos sistemas de alimentación en la respuesta productiva y reproductiva de cuyes (Cavia porcellus). Rev Inv Vet Perú 30: 15151526. doi: 10.15381/rivep.v30i4.17173

16. Sarria J. 2014. El cuy: crianza tecnificada. Manual Técnico en Cuyicultura $\mathrm{N},{ }^{\circ} 1$. Lima, Perú: Univ. Nacional Agraria La Molina. $65 \mathrm{p}$.

17. Solorzano J. 2014. Evaluación de tres sistemas de alimentación comercial de cuyes (Cavia porcellus) en la etapa de reproducción. Tesis de Ingeniero Zootecnista: Lima, Perú: Univ. Nacional Agraria La Molina. 124 p. 